\title{
The Constraint of a General Effective Potential in Vector Torsion Coupled Conformally Induced Gravity
}

\author{
Jewan Kim*, C. J. Park ${ }^{\dagger}$ and Yongsung Yoon $\#$ \\ *Department of Physics, Seoul National University Seoul 151-742, Korea \\ ${ }^{\dagger}$ Institute for Mathematical Science, Yonsei University Seoul 120-749, Korea \\ \# Department of Physics, Hanyang University Seoul 133-791, Korea
}

\begin{abstract}
It is found that the deviation of an effective potential from the quartic form is related to the metric and vector torsion dependencies of the effective potential in the vector torsion coupled conformally induced gravity.
\end{abstract}

\section{INTRODUCTION}

It is considerable that gravity is characterized by a dimensionless coupling constant $\xi$ and that the gravitational constant $G_{N}$ is given by the inverse square of the vacuum expectation value of the dilaton field [1, 2]. The weakness of gravity can be associated with spontaneous symmetry breaking at very high energy scale. The induced gravity action in Riemann space is given by

$$
S_{e f f}\left(\phi ; g_{\beta \gamma}\right)=\int d^{4} x \sqrt{g}\left\{-\frac{1}{2} \xi \phi^{2} R(\{\})+\frac{1}{2} \partial_{\mu} \phi \partial^{\mu} \phi-V\left(\phi ; g_{\beta \gamma}\right)\right\} .
$$

The effective potential $V_{e f f}\left(\phi ; g_{\beta \gamma}\right)$ for the dilaton field may be attributed to quantum fluctuations of the conformal factor or gauge fields coupled to the dilaton field [3 6 . In any case, it is assumed that the effective potential attains its minimum value at $\phi=\sigma$, then the above induced gravity action is reduced to the well known Einstein-Hilbert action with the gravitational constant,

$$
G_{N}=\frac{1}{8 \pi \xi \sigma^{2}}
$$

On the analogy of the $S U(2) \times U(1)$ symmetry of the weak interactions, we can consider a continuous symmetry which is broken through a spontaneous symmetry breaking in the gravitational interactions. The most attractive symmetry is the local conformal symmetry which rejects the Einstein-Hilbert action, but admits the induced gravity action (11) with the specific coupling $\xi=-\frac{1}{6}$ and the bare quartic potential. If we extend Riemann space to the minimal Riemann-Cartan space which has almost Riemannian structure with the additional vector torsion like a conformal gauge field [7], the conformally induced gravity action can be written as follows [2];

$$
\begin{gathered}
S_{e f f}\left(\phi ; S_{\alpha}, g_{\beta \gamma}\right)=\int d^{4} x \sqrt{g}\left\{-\frac{\xi}{2} R(\{\}) \phi^{2}+\frac{1}{2} \partial_{\alpha} \phi \partial^{\alpha} \phi\right. \\
\left.-\frac{1}{4} H_{\alpha \beta} H^{\alpha \beta}+\frac{1}{2}(1+6 \xi) S^{\alpha} \partial_{\alpha} \phi^{2}+\frac{1}{2}(1+6 \xi) S_{\alpha} S^{\alpha} \phi^{2}-V_{e f f}\left(\phi ; S_{\alpha}, g_{\beta \gamma}\right)\right\},
\end{gathered}
$$

where $S_{\mu}=\frac{1}{3} T_{\nu \mu}^{\nu}$ is the vector torsion and $H_{\mu \nu}=\partial_{\mu} S_{\nu}-\partial_{\nu} S_{\mu}$ is the conformal field strength. This action (3) is conformally invariant for arbitrary $\xi$ with the bare quartic potential $\frac{\lambda}{4 !} \phi^{4}$. However, the effective potential $V_{e f f}\left(\phi ; S_{\alpha}, g_{\beta \gamma}\right)$ radiatively corrected by quantum fluctuations depends on the metric in general and breaks the local conformal symmetry. The radiative corrections of the bare quartic potential can give non-trivial minima and nonvanishing vacuum expectation value $\sigma$ to the dilaton field. This symmetry breaking in conformally induced gravity might be applied to the induced gravity inflationary models [8 10].

The quantum effects of the conformal factor for the dilaton field in conformally non-invariant induced gravity with torsion background have been considered in Ref. 11] 14. In the symmetry broken phase, the torsion vector gets the mass $\sqrt{(1+6 \xi)} \sigma$, and the ratio of vector torsion mass $M_{s}$ and planck mass $M_{p}$ is

$$
\frac{M_{s}}{M_{p}}=\sqrt{\frac{(1+6 \xi)}{8 \pi \xi}}
$$

which is independent of the vacuum expectation value $\sigma$ of the dilaton field. Therefore, if $\sqrt{(1+6 \xi)}$ is small enough, it can be considered an approximate quantum gravity such that we treat the metric as a classical background and the vector torsion as a quantum field in the below plank energy scale 15$]$. 


\section{EQUATIONS OF MOTION FOR CONFORMALLY INDUCED GRAVITY}

In this section, we analyze the classical equations of motion for the action Eq.(3). Varying the action, we obtain the following three equations of motion;

$$
\begin{gathered}
\square \phi=-\xi R(\{\}) \phi+(1+6 \xi) \phi\left(S^{\mu} S_{\mu}-\nabla_{\mu} S^{\mu}\right)-\frac{\partial V_{e f f}\left(\phi ; S_{\alpha}, g_{\beta \gamma}\right)}{\partial \phi}, \\
\partial_{\mu}\left(\sqrt{g} H^{\mu \nu}\right)=-(1+6 \xi) \sqrt{g}\left\{\left(\partial^{\nu} \phi\right) \phi+S^{\nu} \phi^{2}\right\}+\frac{\partial V_{e f f}\left(\phi ; S_{\alpha}, g_{\beta \gamma}\right)}{S_{\nu}}, \\
\xi \phi^{2} G_{\mu \nu}=-\left(H_{\mu \alpha} H_{\nu}^{\alpha}-\frac{1}{4} g_{\mu \nu} H_{\alpha \beta} H^{\alpha \beta}\right)+\left(\partial_{\mu} \phi \partial_{\nu} \phi-\frac{1}{2} g_{\mu \nu} \partial_{\alpha} \phi \partial^{\alpha} \phi\right)+(1+6 \xi) \phi^{2}\left(S_{\mu} S_{\nu}-\frac{1}{2} g_{\mu \nu} S_{\alpha} S^{\alpha}\right) \\
+(1+6 \xi)\left(S_{\mu} \phi \partial_{\nu} \phi+S_{\nu} \phi \partial_{\mu} \phi-g_{\mu \nu} S^{\alpha} \phi \partial_{\alpha} \phi\right)+\xi\left\{\nabla_{\mu}\left(\phi \partial_{\nu} \phi\right)+\nabla_{\nu}\left(\phi \partial_{\mu} \phi\right)-g_{\mu \nu} \square \phi^{2}\right\} \\
+g_{\mu \nu} V_{e f f}\left(\phi ; S_{\alpha}, g_{\beta \gamma}\right)-2 \frac{\partial V_{e f f}\left(\phi ; S_{\alpha}, g_{\beta \gamma}\right)}{\partial g^{\mu \nu}}
\end{gathered}
$$

Taking the divergence of Eq.(6), we obtain

$$
(1+6 \xi) \nabla_{\mu}\left(S^{\mu} \phi^{2}\right)=-\frac{1}{2}(1+6 \xi) \square \phi^{2}+\nabla_{\nu} \frac{\partial V_{e f f}\left(\phi ; S_{\alpha}, g_{\beta \gamma}\right)}{\partial S_{\nu}} .
$$

The trace of Einstein Eq.(7) is

$$
-\xi R(\{\}) \phi^{2}=-\partial_{\alpha} \phi \partial^{\alpha} \phi-(1+6 \xi)\left(S^{\alpha} \partial_{\alpha} \phi^{2}+S_{\alpha} S^{\alpha} \phi^{2}\right)-3 \xi \square \phi^{2}+4 V_{e f f}\left(\phi ; S_{\alpha}, g_{\beta \gamma}\right)-2 \frac{\partial V_{e f f}\left(\phi ; S_{\alpha}, g_{\beta \gamma}\right)}{\partial g^{\mu \nu}} g^{\mu \nu} .
$$

From Eqs.(5) and (9), we have

$$
\begin{gathered}
\phi \square \phi+\partial_{\alpha} \phi \partial^{\alpha} \phi+(1+6 \xi) \nabla_{\alpha}\left(S^{\alpha} \phi^{2}\right)+3 \xi \square \phi^{2}= \\
4 V_{e f f}\left(\phi ; S_{\alpha}, g_{\beta \gamma}\right)-\phi \frac{\partial V_{e f f}\left(\phi ; S_{\alpha}, g_{\beta \gamma}\right)}{\partial \phi}-2 \frac{\partial V_{e f f}\left(\phi ; S_{\alpha}, g_{\beta \gamma}\right)}{\partial g^{\mu \nu}} g^{\mu \nu} .
\end{gathered}
$$

Using Eq.(8), we have a $\xi$ independent equation for a general effective potential from Eq.(10) as follows;

$$
4 V_{e f f}\left(\phi ; S_{\alpha}, g_{\beta \gamma}\right)-\phi \frac{\partial V_{e f f}\left(\phi ; S_{\alpha}, g_{\beta \gamma}\right)}{\partial \phi}=2 \frac{\partial V_{e f f}\left(\phi ; S_{\alpha}, g_{\beta \gamma}\right)}{\partial g^{\mu \nu}} g^{\mu \nu}+\nabla_{\nu} \frac{\partial V_{e f f}\left(\phi ; S_{\alpha}, g_{\beta \gamma}\right)}{\partial S_{\nu}} .
$$

Therefore the metric and vector torsion dependencies of an effective potential are directly related to the deviation of the effective potential from the quartic form. Needless to say, if we ignore the metric and vector torsion dependencies of the effective potential, then we encounter inconsistency of the classical equations of motion in case of the vector torsion coupled induced gravity. Because the relation (11) also appears in case of the special coupling $\xi=-1 / 6$, where the vector torsion is decoupled from the dilaton, we can say that the Eq. 11 generally appears in conformally induced gravity model.

The equation (11) also requires that the metric independent bare effective potential should be quartic in the dilaton field. Because this constraint comes from the assumption that the bare action is conformally invariant except the potential term, if we consider non-conformal coupling in kinetic and interacting terms, there would be no such a constraint.

It would be interesting if it can be explicitly realized that the effective potential including the quantum fluctuations of the vector torsion and the dilaton fields with non-vanishing vector torsion background satisfies the Eq.(11) and shows a relevant phase transition in general background space-time leading to an inflationary phase through the conformal symmetry breaking.

Acknowledgments: This work was supported in part by the Korea Science and Engineering Foundation and the Ministry of Education through BSRI-2441 
[1] A. Zee, Phys. Rev. Lett. 42, 417 (1979).

[2] L. Smolin, Nucl. Phys. B 160, 253 (1979).

[3] R. Percacci, The Effective Potential for the Conformal Factor in the Standard Model and Beyond, (Preprint YK.93-09-130, YU.93-2300, August 1993).

[4] S.D. Odintsov, R. Percacci, Renormalization Group Effects in the Conformal Sector of 4D Quantum Gravity with Matter, (Preprint SISSA 45-94-EP, April 1994).

[5] I.L. Shapiro, Hilbert-Einstein Action from Induced Gravity Coupled with Scalar Field, (Preprint HUPD-9407, March 1994).

[6] I.L.Buchbinder, S.D. Odintsov and I.L. Shapiro, "Effective action in Quantum Gravity (Bristol and Philadelphia, Institute of Physics Publishing, 1992)

[7] H.T. Nieh and M.L. Yan, Ann. Phys. (N.Y.) 138, 237 (1982).

[8] D.La and P.J. Steinhardt, Phys. Rev. Lett. 62, 376 (1989), D.La, P.J. Steinhardt and W. Bertschinger, Phys. Lett B 231, 231 (1989), D. La, Phys. Rev D 44, 1680 (1991).

[9] F.S. Accetta, D.J. Zoller and M. Turner Phys. Rev. D 31, 3046 (1985).

[10] D.I. Kaiser, Constraints in the Context of Induced-gravity Inflation, (Preprint DART-HEP 93/06, February 1994).

[11] I. Antoniadis and S.D. Odintsov, Mod. Phys. Lett. A 8, 979 (1993).

[12] E. Elizalde and S.D. Odintsov, Phys. Lett. B 315, 245 (1993).

[13] I.L. Buchbinder, S.D. Odintsov and I.L. Shapiro, Phys. Lett. B 162, 92 (1985).

14] A. Bytsenko, E. Elizalde and S.D. Odintsov, Prog. Theor. Phys. 90, 677 (1993).

[15] J. Kim, C.J. Park and Y. Yoon, Phase Transition in Conformally Induced Gravity with Torsion, to be appeared in Phys. Rev D. 